ISBN 978-93-84422-76-9

6th International Conference on Developments in Engineering and Technology

(ICDET-2017)

Bangkok (Thailand) Feb.6-7, 2017

\title{
Global Chaos Synchronization for Nonlinear Control Systems
}

\author{
Grienggrai Rajchakit \\ Department of Mathematics, Faculty of Science, Maejo University, \\ Chiang Mai 50290, Thailand \\ kreangkri@mju.ac.th
}

\begin{abstract}
The general method for proving the existence of homoclinic trajectories in dissipative systems is developed. The applications of this method to Lorenz-like systems: Lorenz, Shimizu-Morioka, Lu and Chen systems are demonstrated. A criterion for the existence of a homoclinic trajectory within a given family of differential equations (Fishing principle) is presented. New numerical algorithm for the approximation of a homoclinic point in parameters space is constructed. The comparison with Kaplan-Yorke and Shilnikov results is made. In this paper, we study Lu's system. First, we control the chaotic behavior of Lu's system to its equilibrium points using linear feedback control and adaptive control method. Finally, we study chaos synchronization of Lu's system by using active control methods.
\end{abstract}

Keywords: Lu's system, Feedback control, Adaptive control, Synchronization.

\section{Introduction}

The homoclinic trajectories are very important in the bifurcation theory and in the study of the passage to chaos [1-8]. The present work deals with the problem: to find conditions that ensures the existence of homoclinic trajectories in dissipative systems. Consider the extension of Tricomi problem [9-14], which can be described in the following way. Chaos in control systems and controlling chaos in dynamical systems have both attracted increasing attention in recent years. A chaotic system has complex dynamical behaviors that possess some special features, such as being extremely sensitive to tiny variations of initial conditions, having bounded trajectories in the phase space. Controlling chaos has focused on the nonlinear systems such as a Lu's system.

Lu's system was first introduced in [2] which is described by

$$
\begin{aligned}
& \dot{x}=a(y-x) \\
& \dot{y}=-x z+c y \\
& \dot{z}=x y-b z
\end{aligned}
$$

Where $x, y, z$ are state variables, $a, b, c$ are positive constants.

The objectives of this paper are as follows. Firstly, to give sufficient conditions of parameters that make equilibrium points of the Lu's system to be asymptotically stable by using linear feedback control and adaptive control methods. Finally, we investigate adaptive synchronization for the Lu's system using active control. 


\section{Synchronization of the Lu's system}

Consider two nonlinear systems:

$$
\begin{aligned}
& \dot{x}=f(t, x) \\
& \dot{y}=g(t, y)+u(t, x, y)
\end{aligned}
$$

Where $x, y \in \mathfrak{R}^{n}, f, g \in C^{r}\left[\mathfrak{R}^{+} \times \mathfrak{R}^{n}, \mathfrak{R}^{n}\right], u \in C^{r}\left[\mathfrak{R}^{+} \times \mathfrak{R}^{n} \times \mathfrak{R}^{n}, \mathfrak{R}^{n}\right], r \geq 1, \mathfrak{R}^{+}$is the set of non-negative real numbers. Assume that (3.1) is the drive system, (3.2) is the response system, and $u(t, x, y)$ is the control vector.

Definition 2.1. Response system and drive system are said to be synchronic if for any initial conditions $x\left(t_{0}\right), y\left(t_{0}\right) \in \mathfrak{R}^{n}$,

$$
\lim _{t \rightarrow+\infty}\|x(t)-y(t)\|=0
$$

\subsection{Synchronization of the Lu's system using active control}

In this section, we assume that there are two Lu's systems such that the drive system (with the subscript 1) drives the response system (with the subscript 2). The drive and response systems are given, respectively, by

$$
\begin{aligned}
& \dot{x}_{1}=a\left(y_{1}-x_{1}\right) \\
& \dot{y}_{1}=-x_{1} z_{1}+c y_{1} \\
& \dot{z}_{1}=x_{1} y_{1}-b z_{1}
\end{aligned}
$$

and

$$
\begin{aligned}
& \dot{x}_{2}=a\left(y_{2}-x_{2}\right)+u_{1} \\
& \dot{y_{2}}=-x_{2} z_{2}+c y_{2}+u_{2} \\
& \dot{z_{2}}=x_{2} y_{2}-b z_{2}+u_{3}
\end{aligned}
$$

We have introduced three control functions $u_{1}(t), u_{2}(t), u_{3}(t)$ in (2.4). Our goal is to determine the control functions $u_{1}(t), u_{2}(t), u_{3}(t)$. We define the errors system as the difference between system (2.3) and the controlled system (2.4). Let us define the state errors between the response system (2.4) and the drive system (2.3) as

$$
\begin{aligned}
& e_{x}=x_{2}-x_{1} \\
& e_{y}=y_{2}-y_{1} \\
& e_{z}=z_{2}-z_{1}
\end{aligned}
$$

By subtracting (2.3) from (2.4) and by using the notation in (2.5), we have

$$
\begin{aligned}
& \dot{e}_{x}=a\left(e_{y}-e_{x}\right)+u_{1}(t) \\
& e_{y}=x_{1} z_{1}-x_{2} z_{2}-c e_{y}+u_{2}(t) \\
& e_{z}=x_{2} y_{2}-x_{1} y_{1}-b e_{z}+u_{3}(t)
\end{aligned}
$$


We define active control functions $u_{1}(t), u_{2}(t), u_{3}(t)$ as follows

$$
\begin{aligned}
& u_{1}(t)=V_{1}(t)-a e_{y} \\
& u_{2}(t)=V_{2}(t)-x_{1} z_{1}+x_{2} z_{2} \\
& u_{3}(t)=V_{3}(t)-x_{2} y_{2}+x_{1} y_{1} .
\end{aligned}
$$

Hence, the error system (2.6) becomes

$$
\begin{aligned}
& \dot{e}_{x}=V_{1}(t)-a e_{x} \\
& \dot{e}_{y}=V_{2}(t)-c e_{y} \\
& \dot{e}_{z}=V_{3}(t)-b e_{z}
\end{aligned}
$$

The error system (2.7) is a linear system with control input $V_{1}(t), V_{2}(t), V_{3}(t)$ as functions of the error $e_{x}, e_{y}, e_{z}$. There are a number of possible choices for the controls $V_{1}(t), V_{2}(t), V_{3}(t)$. We choose

$$
\left[\begin{array}{l}
V_{1}(t) \\
V_{2}(t) \\
V_{3}(t)
\end{array}\right]=A\left[\begin{array}{l}
e_{x} \\
e_{y} \\
e_{z}
\end{array}\right],
$$

Where $A$ is a $3 \times 3$ constant matrix. Let the matrix $A$ is chosen in the following form

$$
A=\left(\begin{array}{ccc}
-a & 0 & 0 \\
0 & -c & 0 \\
0 & 0 & -b
\end{array}\right)
$$

Since eigenvalues of $A$ are $-a,-b,-c$, where $a, b, c$ are positive constants. It follows that $e_{x}, e_{y}, e_{z}$ converge to zero as $t$ tends to $+\infty$ and hence the Lu's systems (2.3) from (2.4) are synchronic.

\section{Numerical Simulations}

Fourth-order Runge-Kutta integration method is used to solve two systems of differential Equations. (2.3) and (2.4) with time step size 0.01 . The values of parameters in (2.3) are chosen as $a=5, b=10, c=0.5$ to ensure the chaotic behavior of Lu's systems. The initial conditions of the drive system are $x_{1}(0)=0.65, y_{1}(0)=0, z_{1}(0)=0$ and the initial conditions of the response system are $x_{2}(0)=0.2, y_{2}(0)=0.1, z_{2}(0)=0.1$. Thus, the initial values of the error system are $e_{x}(0)=-0.69, e_{y}=0.25, e_{z}=0.15$. Fig. 1-3 show that the synchronization is occurred after applying active control at $t=10$. Fig. 4 show that the state errors $\left(e_{x}, e_{y}, e_{z}\right)$ of Lu's systems of equations with the active control activated.

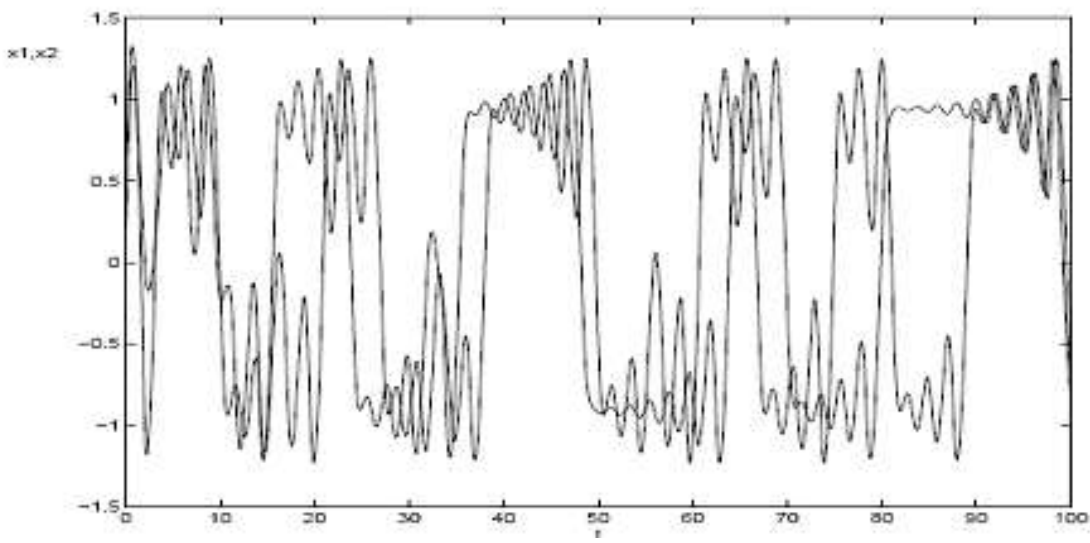

Fig. 1 The state $x_{1}, x_{2}$ of the coupled Lu's systems of equations with the active control activated. 


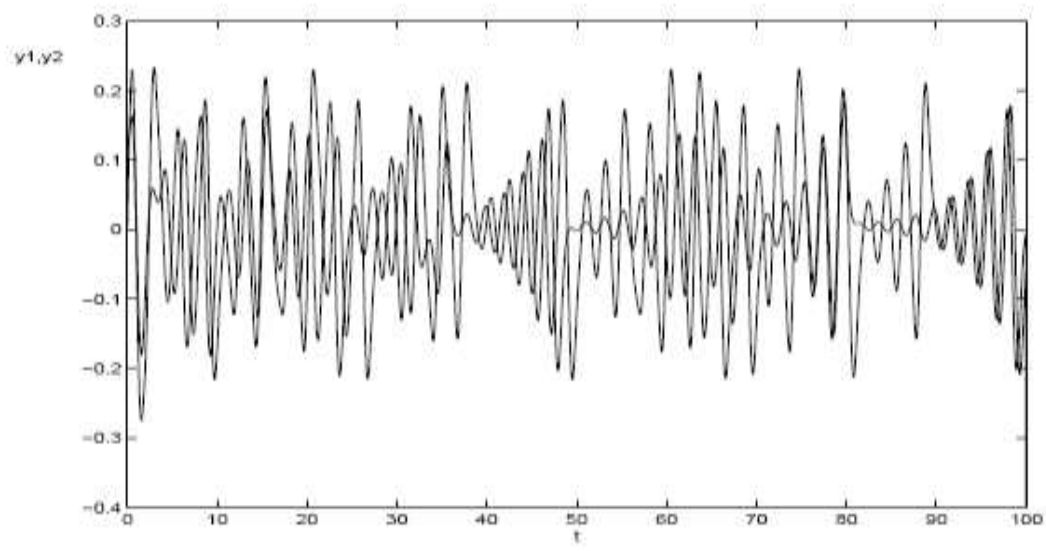

Fig. 2 The state $y_{1}, y_{2}$ of the coupled Lu's systems of equations with the active control activated.

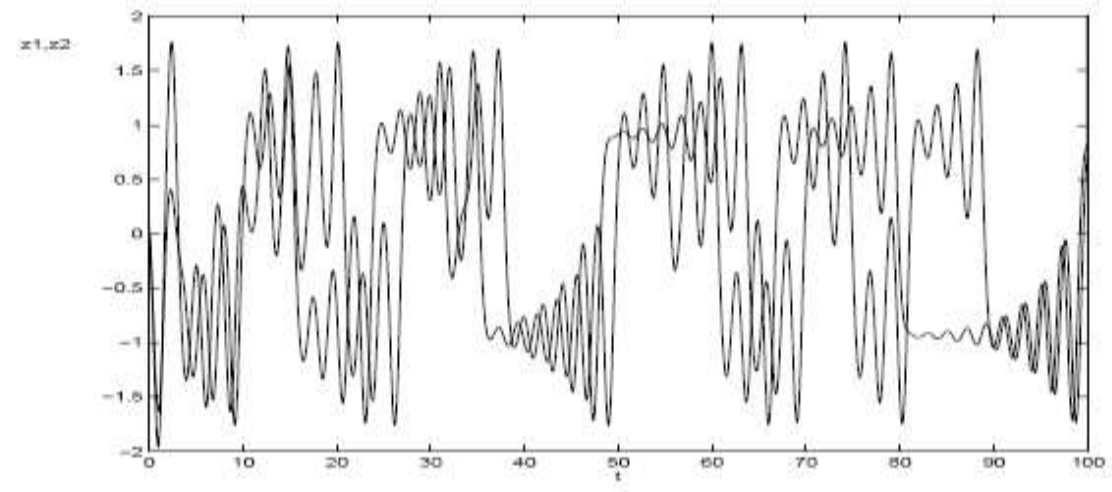

Fig. 3 The state $z_{1}, z_{2}$ of the coupled Lu's systems of equations with the active control activated.

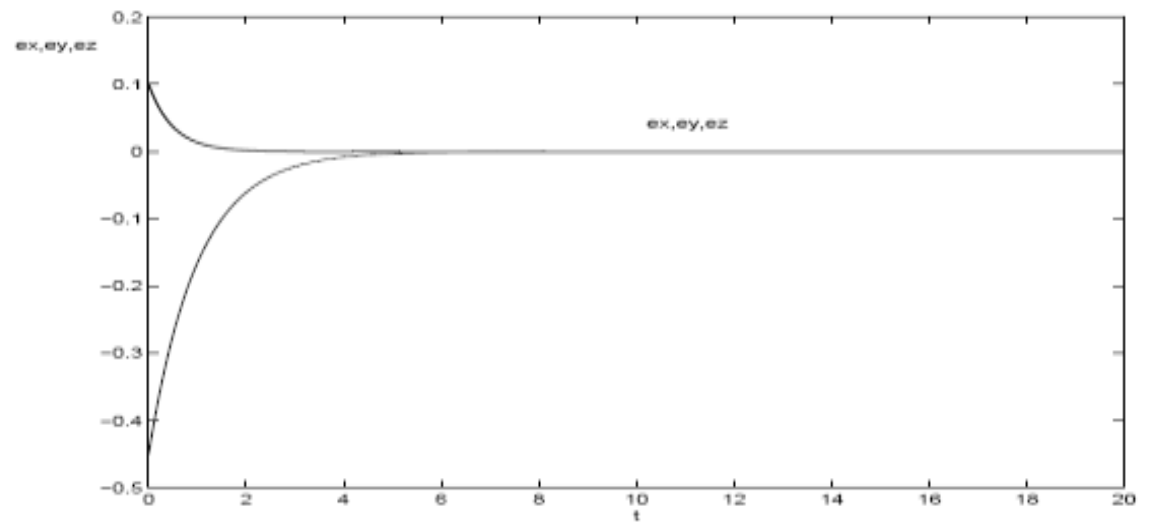

Fig. 4 The state errors $\left(e_{x}, e_{y}, e_{z}\right)$ of the coupled Lu's systems of equations with the active control activated.

\section{Conclusions}

In this paper, we first give sufficient conditions for stability of equilibrium points of linear feedback controls which control the chaotic behavior of Lu's system to its equilibrium points. Finally, we give active controls which synchronize Lu's system. Numerical Simulations are also given to verify results we obtained. 


\section{Acknowledgements}

This work was supported by the Office of Agricultural Research and Extension Maejo University Chiangmai Thailand, the Thailand Research Fund Grant(RSA5980019), the Higher Education Commission and Faculty of Science, Maejo University, Thailand.

\section{References}

[1] K. Abdelwahab and R.B. Guenther, An introduction to numerical methods a MATLAB approach, Chapman and Hall/CRC, New York, 2002.

[2] S. Barnett and R.G. Cameron, Introduction to Mathematical Control Theory, Oxford, Clarendon Press, 1985.

[3] J. Lu, G. Chen, A new chaotic attractor coined, Int. J. Bifurc. Chaos 12 (2002) 659-661.

https://doi.org/10.1142/S0218127402004620

[4] V.N. Phat, Constrained Control Problems of Discrete Processes, World Scientific Publisher, Singapore-NewJerseyLondon,1996.

[5] V.N. Phat, Introduction to Mathematical Control Theory, Hanoi National University Publisher, Hanoi, 2001.

[6] K. Ratchagit, "Asymptotic Stability of Delay Difference System of the Hopfield Neural Networks via Matrix Inequalities and Application", International Journal of Neural Systems, Vol. 17, No. 5, pp. 425-430 (2007).

https://doi.org/10.1142/S0129065707001263

[7] K. Ratchagit, "Asymptotic Stability of Delay-Difference Control System of Hopfield Neural Networks via Matrix Inequalities", International Mathematical Forum, Vol. 4, No.28, pp. 1405-1411 (2009).

[8] K. Ratchagit, "Asymptotic Stability of Time Varying Delay-Difference System of Cellular Neural Networks with TimeVarying Delay via Matrix Inequalities and Applications", Int. J. of Appl. Math and Mech, Vol. 5, No. 3, pp. 11-21 (2009).

[9] K. Ratchagit, "Asymptotic Stability of Nonlinear Delay-Difference Control System with Time-Varying Delay via Matrix Inequalities", Applied Mathematical Sciences, Vol. 3, No.30, pp. 1473-1478 (2009).

[10] K. Ratchagit, M. Ratchagit, in: International Conference on Circuits, System and Simulation, IPCSIT, vol. 7, 2011, pp. 188-192.

[11] F. Zhang, C. Mu, X. Li, International Journal of Bifurcations and Chaos 22 (1) (2012).

[12] I. Li, G. Chen, International Journal of Bifurcation and Chaos 16 (10) (2006) 3035. https://doi.org/10.1142/S021812740601663X

[13] V.A. Yakubovich, G.A. Leonov, A.Kh. Gelig, Stability of Stationary Sets in Control Systems with Discontinuous Nonlinearities, World-Scientific, 2004, 334 pp.

https://doi.org/10.1142/5442

[14] G.A. Leonov, Journal of Applied Mathematics and Mechanics 74 (2) (2010) 135.

https://doi.org/10.1016/j.jappmathmech.2010.05.002 